\title{
Borderline Ovarian Mucinous Tumor/Atypical Proliferative Ovarian Mucinous Tumor
}

National Cancer Institute

\section{Source}

National Cancer Institute. Borderline Ovarian Mucinous Tumor/Atypical Proliferative

Ovarian Mucinous Tumor. NCI Thesaurus. Code C122586.

A non-invasive neoplasm that arises from the ovary and is composed of gastrointestinaltype, mucin-containing epithelial cells. It shows greater cellular proliferation and cytologic atypia as compared to benign ovarian mucinous tumors. 